\title{
Ignition of Combustible Dust Layers on a Hot Surface
}

\author{
C. C. HWANG
}

Department of Mechanical Engineering

University of Pittsburgh

Pittsburgh, Pennsylvania 15261, USA

\section{D. LITTON}

Pittsburgh Research Center

U.S. Bureau of Mines

Pittsburgh, Pennsylvania 15236, USA

\begin{abstract}
This paper studies theoretically the heating and possible ignition of a layer of combustible dust. on a hot surface. A new approach to this problem is the use of thermal conductivity equations for gramular materials which have been developed by previous workers. The transient heat conduction equation with temperature-dependent distributed heat sources is solved numerically. In addition to the case of negligible reactant consumption, effects of reactant consumption on the heating behavior in dust layers are also considered. The results of computations are compared with experimeital results obtained by other workers.
\end{abstract}

KEYWORDS: ignition, combustion, dust

\section{INTRODUCTION}

A combustible dust layer on a hot surface may ignite if the temperature of the surface is sufficiently high. To prevent the ignition of a dust layer and the resulting fire and explosion, it is necessary to determine the minimum surface temperature and controlling parameters for ignition of a particular type of dust.

One way to determine experimentally the minimum dust layer ignition temperature is to deposit a dust layer of given size and thickness on a horizontal circular plate heated to a predetermined temperature. The upper surface of the layer is open to the atmosphere. Experiments are repeated, with fresh layers at different temperatures, until the critical temperature for ignition is found. The National Academy of Sciences (NAS) Committee on Evaluation of Industrial Hazards has recommended a hotplate test to determine minimum hot- surface ignition temperatures of dust layers [1]. According to NAS recommendations, ignition in the dust is considered to have occurred at the minimum hot plate temperature at which any of the following occurs:

1. There is visible evidence of combustion such as red glow or flame; 
2. The slope of the temperature-time curve for a thermocouple in the center of the dust layer continues to increase;

3. A temperature rise of $50^{\circ} \mathrm{C}$ above the hotplate temperature occures in the dust;

4. The dust melts.

Palmer and Tonkin $[2]$ measured the ignition temperature of layers of mixed wood sawdust and coal dust on a hot surface. Bowes and Townshend [3] determined experimentally the minimum temperatures for ignition of beech sawdust on a hot surface. The results indicated that the depth of the layer is the most important factor affecting the minimum temperature of the hot surface for ignition. Particle size was found to be not important over a wide range, and the packing density affected the ignition temperature of thin layers only. The experimental results were correlated by a thermal theory of ignition based on a simple model of exothermic reaction.

Miron and Lazzara [4] used a hotplate and a test procedure recommended by the National Acadiemy of Sciences to determine minimum dust layer ignition temperatures for several dusts. The dusts included coal, three oil shales, lycopodium spores, corn starch, grain and brass powder. Hot-surface ignition temperature increased with (i) decreasing layer thickness, (ii) increasing particle size, and (iii) decreasing oil shale grade.

The objective of the present study is to model mathematically the processes of heating leading to possible ignition in a dust layer which is placed on top of a constanttemperature heated surface. A new feature in the study is the use of a thermal conductivity model for granular materials. This model accounts for the conduction and thermal radiation in local cell volumes. In addition to the case of negligible reactant consumption, effects of reactant consumption on the heating behavior in dust layers are also considered.

\section{MATHEMATICAL FORMULATION}

\section{Some Experimental Observations}

The temperature-time profiles as recorded by thermocouples in hot-plate tests of dust layers $[3,4]$ show 4 trends of heating characteristics:

1. The temperature increases monotonically toward the plate surface temperature. The temperature, after reaching a plateau which is not more than $50^{\circ} \mathrm{C}$ above the plate temperature, decreases monotonically. No ignition takes place for this case.

2. The temperature increases monotonically toward the plate surface temperature. After reaching a plateau, the increase in temperature accelerates rapidly, indicating ignition.

3. The temperature increases monotonically toward the plate surface temperature. Without leveling off, the temperature continues to increase above the plate temper. ature, indicating ignition. 
4. The temperature increases in the beginning. Instead of reaching a plateau prior to the accelerating stage, the temperature profile goes through a pronounced trough. The temperature then increases more than $50^{\circ} \mathrm{C}$ above the plate temperature, indicating ignition.

The trends of dust layer heating described above indicate that many physical and chemical processes take place simultaneously. These inclucle thermal decomposition, oxidation and other chemical reactions, in addition to all modes of heat transfer. The dust may ignite prior to substantial reactant consumption. In some cases, the consumption of reactant may not be neglected. It has been observed [4] that softening, fusing, and charring of media may occur, resulting in sagging, collapsing, and swelling of the layer.

The present paper will not attempt to model all the phenomena observed in experiments. Rather, a simple model which can reasonably represent major features of the thermal processes observed will be employed. It may be noted that kinetic data and some physical properties are not readily available even if an elaborate model can be constructed.

\section{Equations for Temperature Distribution}

A dust layer will be considered as an infinite plane slab of thickness, L, initially at a uniform temperature equal to the surrounding temperature $T_{\infty}$. At time $t=0$, one face of the slab is brought into perfect thermal contact with a plane surface at a constant temperature $T_{p}$ greater than $T_{0}$. Heat is lost by convection and radiation from the other face.

The dust is considered to be consisted of active material and of inert material. For instance, for wood a typical virgin material density, $\rho_{s}$, is $500 \mathrm{~kg} / \mathrm{m}^{3}$ and the char density, $\left(\rho_{s}-\rho_{a 0}\right)$, is $125 \mathrm{~kg} / \mathrm{m}^{3}[5]$. This signifies that the active material is $75 \%$ and the inert material is $25 \%$. For substances like nitrocellulose the active material is $100 \%$. As the dust reacts due to an increase in the local temperature, the active material is continuously converted into volatiles and heat is generated. Let $\rho_{a}(x, t)$ be the timedependent density of the reacted active material. Initially, the active material density is $\rho_{a 0}$ and the virgin dust material density is $\rho_{s}$. Thus, the inert material density is $\left(\rho_{s}-\rho_{a 0}\right)$. The rate of conversion of the active material is assumed to be governed by a sinple first-order Arrhenius decomposition reaction, that is

$$
\frac{d \rho_{a}}{d t}=\left(\rho_{a 0}-\rho_{a}\right) A \exp \left(-\frac{E}{R T}\right)
$$

Let $\omega\left(=\rho_{a} / \rho_{\alpha 0}\right)$ be the fraction of the active material reacted. Then the above equation can be written as

$$
\frac{d \omega}{d t}=(1-\omega) A \exp \left(-\frac{E}{R T}\right)
$$

The temperature distribution in the dust layer is given by the one- dimensional equation of heat conduction

$$
\rho c \frac{\partial T}{\partial t}=\frac{\partial}{\partial x} k \frac{\partial T}{\partial x}+Q \rho_{a 0} \frac{d \omega}{d t} .
$$


Taking $x$ as zero at the hot-plate surface and $L$ as the cool surface, the boundary conditions are:

$$
\begin{aligned}
T & =T_{p}, \quad x=0 \\
-k \frac{\partial T}{\partial x} & =h\left(T-T_{\infty}\right)+\epsilon \sigma\left(T^{4}-T_{\infty}^{4}\right),
\end{aligned}
$$

where $\epsilon$ is the emissivity of the layer surface, $\sigma$ is the Stefan-Boltzman constant, and $h$ is the convective heat transfer coefficient, the value of which is determined from [6]

$$
\bar{N} u_{D}=0.54 R a_{D}^{1 / 4}
$$

The initial condition is

$$
T=T_{\infty}, \quad t=0, \quad 0 \leq x \leq L .
$$

For the case of negligible reactant consumption, Equation (2) is not required, and Equation (3) is written as

$$
\rho c \frac{\partial T}{\partial t}=\frac{\partial}{\partial x} k \frac{\partial T}{\partial x}+Q \rho A \exp \left(-\frac{E}{R T}\right) .
$$

The same boundary conditions (4), (5) and the initial condition (7) apply,

Similar equations to describe the processes of thermal ignition $[7,8,9]$ and pyrolysis [5] have been given by previous workers.

\subsection{Thermal Conductivity for Dust Layer}

It is customary to calculate heat transfer in dust lavers by the use of a single "overall thermal conductance", $k_{e}$. Since heat transfer in dust lavers can occur by conduction, convection, and thermal radiation, the quantity $k_{e}$ is generally different from the local thermal conductivity $k$. Zumbrunnen, et al. [10] developed an expression for the local thermal conductivity, $k$, in packed beds. Heat transfer mechanisms by conduction and radiation through various paths in packed beds were considered. It was assumed that particle surfaces are opaque, diffuse-gray; the air is radiatively nonparticipating; and voids between particles are blackbody cavities. The local thermal conductivity is given by

$$
k=\psi d\left[(1-\Lambda-\delta)\left(\frac{\psi d}{k_{s}}+\frac{1}{h_{r s}+\frac{k_{f}}{\phi d}}\right)^{-1}+\Lambda h_{r v}\right]+\delta k_{s},
$$

where the heat transfer "coefficients" for thermal radiation are

$$
h_{r s}=4 \sigma \bar{T}^{3}\left(\frac{\epsilon}{2-\epsilon}\right)
$$

and

$$
h_{r v}=4 \sigma \tilde{T}^{3}\left[1+\frac{\Lambda}{2(1-\Lambda)}\left(\frac{1-\epsilon}{\epsilon}\right)\right],
$$


where $\bar{T}$ is the average temperature of the surface enclosing the voids.

The dimensionless parameter $\phi$ is the ratio of the effective length for conduction across the air to the characteristic particle size. Zumbrunnen, et al. [10] found $\phi=0.092$ by adjusting the value until the prediction agreed with their experimental data. The parameter $\psi$ is the ratio of the effective length $\ell_{s}$ for conduction across a particle to the characteristic particle size $d$ and is always less than unity. Their experiments showed that this value is on the order of 0.8 . The parameter $\Lambda$ is related to the bed porosity, $p$, by

$$
\Lambda=1-(1-p)^{2 / 3} .
$$

A parametric study of Equation (9) and comparisons of measured and predicted thermal conductivities have been made by Zumbrunnen, et al. [10]. In the present computations the values of $\phi$ and $\psi$ are taken to be 0.092 , and 0.8 , which are determined by Zumbrunnen, et al. The porosity $p$ is determined from the effective layer density (measured at the beginning of tests) and the virgin dust density. Yagi and Kunii [11] reported that the values of $\delta$ are less than 0.00001 . A value of $\delta=0.1 \times 10^{-3}$ is used in the present computations, assuming that when the materials are consumed during reaction the particles tend to shift and increase their contact areas.

\section{RESULTS}

Equations (2) and (3), together with Equations (4) through (7), are solved numerically by a finite difference technique. For the case of negligible reactant consumption, Equation (8) together with Equations (4) through (7) are solved.

Figure 1 shows the temperature distributions in a $25 \mathrm{~mm}$ beech sawdust layer at different instants of time. In the first stage of heating, the sawdust layer behaves as an inert substance. In the next stage of heating, the heat generation starts to show its effect, especially in the vicinity of the hot surface. Approximately 90 minutes after the commencement of the heating, the temperature of dust near the hot plate rises above that of the hot plate, indicating that the heat transfer takes place from the sawdust to the plate. The temperature distribution shows a bulge which accelerates in its growth, and eventually the temperature at some point becomes extremely high. This behavior of heating has been called a "thermal runaway", and may be interpreted as the ignition of the sawdust. Computationally, an overflow in a numerical value will result. To prevent the overflow, the time step in the computation must be decreased. For instance, the time step, $\Delta t$, has been made inversely proportional to the maximum temperature increase in one time step, $\triangle T$, a.t a point in the layer.

Plots of temperature-time profiles at different points $(x=5,7.5$, and $12.5 \mathrm{~mm})$ in the $25 \mathrm{~mm}$-thick beech sawdust layer are shown in Figure 2. The experimental results are for $x=10 \mathrm{~mm}$ in a $25 \mathrm{~mm}$ beech sawdust layer on a hot surface at $548 \mathrm{~K}$ [3]. The experimental data indicate ignition approximately at 80 minutes whereas the computations do not show accelerated temperature rise until approximately 100 minutes. It may be noted that the kinetic data as determined based on the steady-state solution are used in the present computations; a better agreement between the experiments and the theory can be obtained by adjusting the kinetic data. 


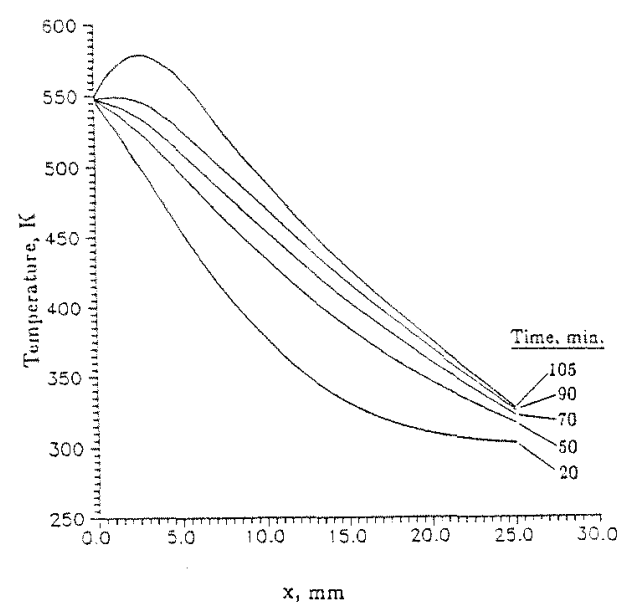

FIGURE 1. Temperature distribution in $25 \mathrm{~mm}$ dust layer at times indicated, $k_{g}=0.14$ $\mathrm{W} / \mathrm{m} \cdot \mathrm{K}, \rho=520 \mathrm{~kg} / \mathrm{m}^{3}, c=2500 \mathrm{~J} / \mathrm{kg} \cdot \mathrm{K}$, $d=0.5 \times 10^{-3} \mathrm{~m}, p=0.6, \epsilon=0.9, \psi=0.8$, $\phi=0.092, \delta=0.1 \times 10^{-3}, D=0.27 \mathrm{~m}, T_{\infty}$ $=300 \mathrm{~K}, T_{p}=548 \mathrm{~K}, Q \rho A=0.393 \times 10^{16}$ $\mathrm{W} / \mathrm{m}^{3}, E=0.108 \times 10^{9} \mathrm{~J} /$ mole. The kinetic data obtained from Bowes and Townshend (1962).

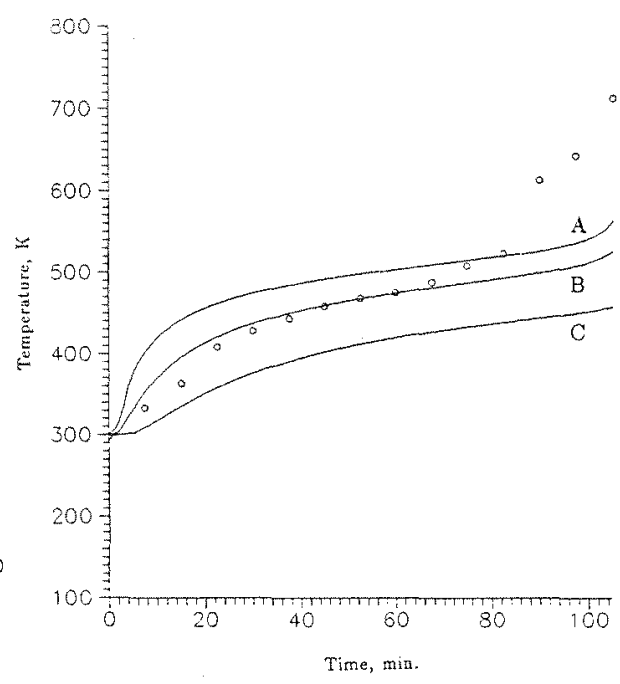

FIGURE 2, Temperature-time profiles for $25 \mathrm{~mm}$ dust layer. (A): $x=5 \mathrm{~mm},(\mathrm{~B}): x=7.5 \mathrm{~mm}$, (C): $x=12.5 \mathrm{~mm}$. O: experimental data using beech sawdust, $x=10 \mathrm{~mm}$ (Bowes and Townshend 1962). Same physical and kinetic data as in Fig. 1.

Figure 3 shows the minimum dust layer ignition temperature as a function of the layer thickness $L$. The same physical and kinetic data as in Figure 1 are used in constructing the curve in Figure 3. The minimum dust layer ignition temperatures are computed for the layer thickness $L=6.35,12.7,25$, and $38.1 \mathrm{~mm}$. The ignition is considered to occur when the adiabatic condition, i.e., $d T / d x=0$, is reached at $x=0$. The experimental data in Figure 3 are taken with $d=0.12-0.85 \mathrm{~mm}$, whereas $d=0.5$ $\mathrm{mm}$ is used in all the computations.

Figure 4 shows temperature distribution in a $12.7 \mathrm{~mm}$ dust layer. The consumption of reactant $\left(\rho_{a 0} / \rho_{s}=0.40\right)$ is included in the computation. The physical and kinetic data are more or less artificial, though the activation energy is close to those of coal [12] and charring materials [13]. The general appearance of the temperature distributions is similar to that in Figure 1, though the bulging at the time of thermal runaway appears to be more localized. The distributions of the fraction of the active material reacted, $\omega$, are shown in Figure 5. For this example, only $8 \%$ of the active material is consumed at $x=$ 0 at the time of ignition. The front of the material reaction moves like a wave front. At the time of ignition, the reaction front reaches $6 \mathrm{~mm}$ from the hot surface.

The temperature-time profiles of Pittsburgh coal dust layer heated by a $0.27 \mathrm{~m}$ diameter plate are reported by Miron and Lazzara [4]. The results of computation for 


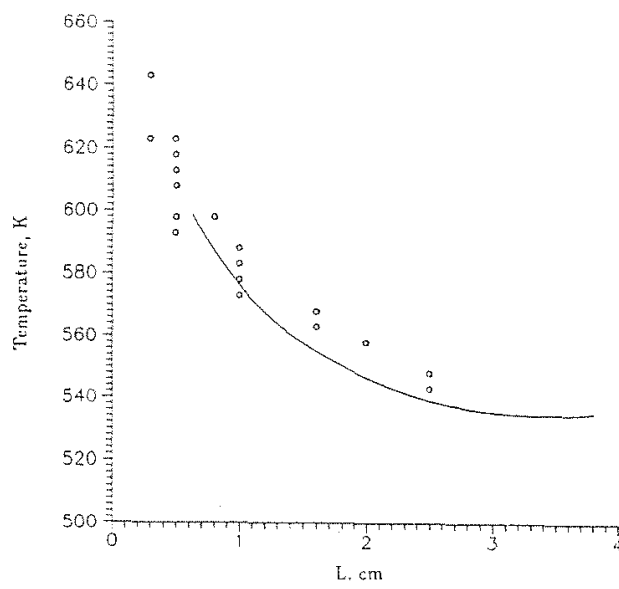

FIGURE 3. Minimum hot-surface ignition temperature as a function of layer thickness. $O$ : experimental data using beech sawdust of various sizes (Bowes and Townshend 1962). Same physical and kinetic data as in Fig. 1.

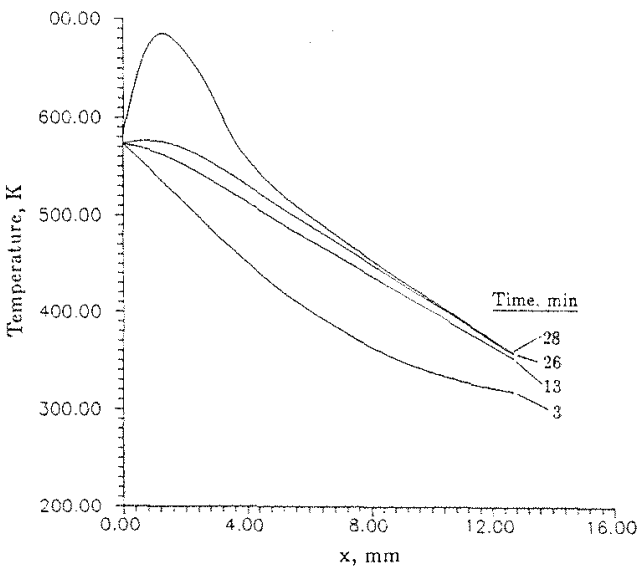

FIGURE 4. Temperature distribution in $12.7 \mathrm{~mm}$ dust layer at times indicated. $k_{*}=0.21$ $\mathrm{W} / \mathrm{m} \cdot \mathrm{K}, p=520 \mathrm{~kg} / \mathrm{m}^{3}, c=947 \mathrm{~J} / \mathrm{kg} \cdot \mathrm{K}, d=$ $0.49 \times 10^{-4} \mathrm{~m}, p=0.592, \epsilon=0.9, \psi=0.8$, $\phi=0.092, \delta=0.1 \times 10^{-3}, D=0.27 \mathrm{~m}, T_{\infty}$ $=300 \mathrm{~K}, T_{\mathrm{p}}=573 \mathrm{~K}, Q=0.65 \times 10^{8} \mathrm{~J} / \mathrm{kg}$, $A=0.25 \times 10^{10} \mathrm{~s}^{-1}, E=0.15 \times 10^{9} \mathrm{~J} / \mathrm{mole}$.

$L=12.7 \mathrm{~mm}$ are shown in Figure 6, along with the experimental data [4]. The value of $E$ is taken as $0.9 \times 10^{8} \mathrm{~J} / \mathrm{kmole}$, which is the value used by Edwards [14] and approximately the average value used by Kansa and Perlee [15] and Davis, et al. [12]. The value of $Q$ used is approximately equal to that of the heating value of Pittsburgh coal; the value of $A$ is adjusted to match the experimental data. Agreement between the computation and experimental result is good up to the thermal runaway in the mathematical model. The temperature in the experiment continues to rise beyond the hotplate temperature of $513 \mathrm{~K}$ and reaches $670 \mathrm{~K}$ before starting to decrease.

\section{DISCUSSIONS AND CONCLUSION}

Heating of combustible dust layers by a hot surface comprises a sequence of complex processes. The one-dimensional equation for conduction of heat with a simple Arrhenius reaction expression for heat generation appears to explain many observed features of the processes. A rapid, boundless increase in the local temperature in the layer may be interpreted as ignition. Temperature-time profiles agree with experimental results when appropriate kinetic data are employed. In particular, computations predict the 


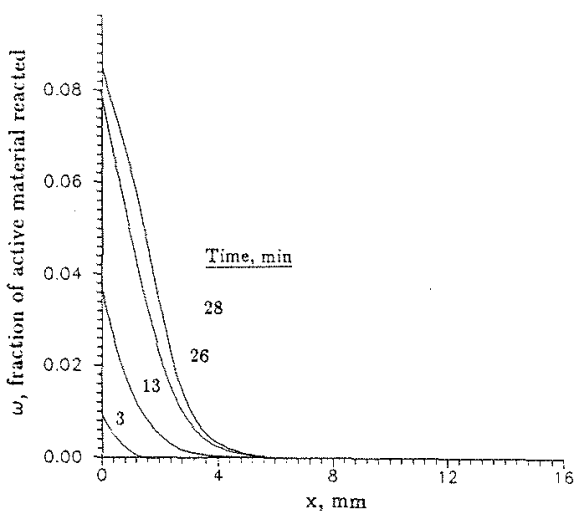

FIGURE 5. Degree of active material reacted in $12.7 \mathrm{~mm}$ dust layer at times indicated. Same physical and kinetic data as in Fig. 4.

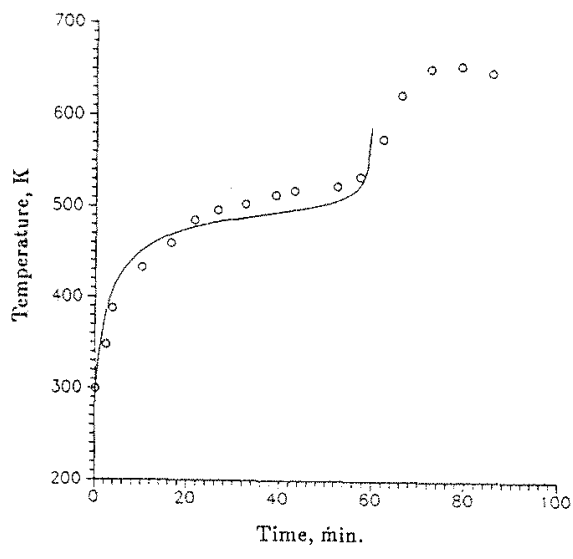

FIGURE 6. Temperature-time profile for $12.7-\mathrm{mm}$ thick layer of Pittsburgh coal. $O$ : experimental data of Miron and Lazzara (1988). - : computations with $x=3.81 \mathrm{~mm}, k_{\text {s }}$ $=0.20 \mathrm{~W} / \mathrm{m} \cdot \mathrm{K}, \rho=1250 \mathrm{~kg} / \mathrm{m}^{3}, \rho_{a 0}=625$ $\mathrm{kg} / \mathrm{m}^{3}, c=1400 \mathrm{~J} / \mathrm{kg} \cdot \mathrm{K}, d=0.30 \times 10^{-4} \mathrm{~m}$, $p=0.5, \epsilon=0.9, \psi=0.8, \phi=0.002, \delta=$ $0.1 \times 10^{-3}, D=0.27 \mathrm{~m}, T_{\infty}=300 \mathrm{~K}, T_{p}=$ $513 \mathrm{~K}, Q=0.365 \times 10^{8} \mathrm{~J} / \mathrm{kg}, A=0.38 \times 10^{5}$ $\mathrm{s}^{-1}, E=0.9 \times 10^{8} \mathrm{~J} / \mathrm{mole}$.

effect of the layer thichness on the minimum dust-layer ignition temperature. One of the observed heating characteristics, i.e., temperature reaching a plateau above the hot-plate temperature without ignition, can be attributed to consumptions of reactants.

Appropriate kinetic data, however, are in general not available in the open literature. Even if the data are available for a particular material, they may have to be adjusted because temperature-time profiles are sensitive to the data, and the conditions under which the data are taken may be quite different from the particular application.

The thermal conductivity for granular material, which is used in the computation, represents a more realistic model than a constant thermal conductivity. Here again, the model suffers from insufficient experimental data. For instance, the change in the particle diameter, effect of internal fluid flow during heating (due to pyrolysis), and change in the solid thermal conductivity of solid are not taken into account.

In the case of concurrent competing processes, such as pyrolysis processes of coal [16], a single Arrhenius expression for reaction is not sufficient to model the processes. The trend 4 described in $\$ 2.1$ appears to be an example of such processes in which a trough appears in a time-temperature profile. During the period when the temperature decreases (trough), the value of $Q$ must be negative. At least two Arrhenius expressions are required to model the processes. In addition, the transport of oxygen to and into the particle may control the rate of reactions. For this case, a diffusion equation must be added to the governing equations. 


\section{NOMENCLATURE}

A: pre-exponential factor, $\mathrm{s}^{-1}$

c: specific heat of dust, $\mathrm{J} / \mathrm{kg} \cdot \mathrm{K}$

$D$ : diameter of hot plate, $m$

$E$ : activation energy, $J / \mathrm{kmole}$

$g$ : acceleration of gravity, $\mathrm{m} / \mathrm{s}^{2}$

$\bar{h}$ : average heat transfer coefficient, $\mathrm{W} / \mathrm{m}^{2} \cdot \mathrm{K}$

$h_{r s}$ : radiative heat transfer coefficient between surfaces of adjacent particles, $\mathrm{W} / \mathrm{m}^{2} \cdot \mathrm{K}$

$h_{r v}$ : radiative heat transfer coefficient between the voids of adjacent particles, $\mathrm{W} / \mathrm{m}^{2} \cdot \mathrm{K}$

$k_{f}$ : thermal conductivity of air, $\mathrm{W} / \mathrm{m} \cdot \mathrm{K}$

$k_{s}$ : thermal conductivity of solid, $\mathrm{W} / \mathrm{m} \cdot \mathrm{K}$

$\ell_{f}$ : effective length for conduction across voids, $m$

$\ell_{s}$ : effective length for conduction through particles, $m$

$\bar{N} u_{D}$ : average Nusselt number, $\bar{h} D / k$

$R$ : universal gas constant, $\mathrm{J} /$ mole $\cdot \mathrm{K}$

$R a_{D}:$ Rayleigh number, $\beta g\left(T_{w}-T_{\infty}\right) D^{3} / \nu \alpha$

$\alpha$ : thermal diffusivity, $\mathrm{m}^{2} / \mathrm{s}$

$\beta:$ coefficient of thermal expansion, $\mathrm{K}^{-1}$

$\delta$ : fraction of area having perfect contact between particles

$\Lambda$ : fraction of area pertaining to voids

$\nu$ : kinematic viscosity, $\mathrm{m}^{2} / \mathrm{s}$

$\phi: \ell_{f} / d$

$\psi: \ell_{s} / d$

\section{REFERENCES}

1. National Academy of Sciences, 1982, Committee on Evaluation of Industrial Hazards, Classification of Combustible Dusts Relative to Electrical Equipment in Class II Hazardous Locations, NMAB 353-4, National Academy Press, Washington, DC.

2. Palmer, K.N. and Tonkin, P.S., 1957, "The Ignition of dust Layers on a Hot surface," Combustion and Flame 1, 14-18. 
3. Bowes, P.C. and Townshend, S.E., 1962, "Ignition of Combustible Dusts on Hot Surfaces," Btit. J. Appl. Phys., 13, 105-114.

4. Miron, Y, and Lazara, C.P., 1988, "Hot-Surface Ignition Temperatures of Dust Layers." Fire and Materials 12, 115-126.

5. Kung, H.C., 1972. "A Mathematical Model of Wood Pyrolysis," Combustion and flame 18, 185-195.

6. Incropera, F.P. and DeWitt, D.P., 1990, "Introduction To Heat Transfer", Second Ed. p.506, John Wiley \& Sons, Inc., New York.

7. Merzhanov, A.G. and Averson, A.E., 1971, "The Present State of the Thermal Ignition Theory: An Invited Review," Combustion and Flame 16 89-124.

8. Thomas, P.H., 1972, "Self-Heating and Thermal Ignition - A Guide to its Theory" and Application," in Ignition, Heat Release, and Noncombustibility of Materials, ASTM STP 502, American Society for Testing and Materials, 56-82.

9. Bowes, P.C., 1984 "Self-heating: Evaluating and Controlling the Hazards", Elsevier, New York.

10. Zumbrunnen, D.A., Viskanta, R., and Incropera, F.P., 1984, "Heat Transfer through Granular Beds at High Temperature," Wärme- und Stoffübertragung 18, 221-226.

11. Yagi, S. and Kunii, D., "Studies on Effective Thermal Conductivities in Packed Beds," AIChE Journal 3 (1957) 373-381.

12. Davis, W.P., Baer, A.D., and Ryan, N.W., 1984, "A Shock-Tube Ignition Study of a Utah Coal," Combustion and Flame 58, 201-215.

13. Sibulkin, M., 1987, "Heat of Gasification for Pyrolysis of Charring Materials," Fire Safety Science - Proceedings of the First International Symposium, 391-400.

14. Edwards, J.C., 1990, "Mathematical Modeling of Spontaneous Heating of a Coalbed," Report of Investigations 9296, Bureau of Mines, U.S. Dept. of the Interior.

15. Kansa, E.J. and Perlee, H.E., 1980, "A Transient Dust-Flame Model: Application to Coal - Dust Flames," Combustion and Flame 38, 17-36.

16. Smoot, L.D. and Pratt, D.T., 1985, "Pulverized - Coal Combustion and Gasification," Chapter 8, Plenum Press, New York. 